\title{
Cervical Cancer and HIV Diseases Co-dynamics with Optimal Control and Cost Effectiveness
}

\author{
Geomira George Sanga ${ }^{1, *}$, Oluwole Daniel Makinde², Estomih Shedrack Massawe ${ }^{1}$, \\ Lucy Namkinga ${ }^{3}$ \\ ${ }^{1}$ Department of Mathematics, University of Dar es Salaam, Dar es Salaam, Tanzania \\ ${ }^{2}$ Faculty of Military Science, Stellenbosch University, Saldanha, South Africa \\ ${ }^{3}$ Department of Molecular Biology and Biotechnology, University of Dar es Salaam, Dar es Salaam, Tanzania
}

Email address:

gmtisi@gmail.com (G. G. Sanga), makinded@gmail.com (O. D. Makinde), estomihmassawe@yahoo.com (E. S. Massawe), odulajalucy@yahoo.com (L. Namkinga)

*Corresponding author

\section{To cite this article:}

Geomira G. Sanga, Oluwole D. Makinde, Estomih S. Massawe, Lucy Namkinga. Cervical Cancer and HIV Diseases Co-dynamics with Optimal Control and Cost Effectiveness. Pure and Applied Mathematics Journal. Vol. 6, No. 4, 2017 pp. 124-136. doi: $10.11648 /$ j.pamj.20170604.14

Received: June 22, 2017; Accepted: July 7, 2017; Published: August 4, 2017

\begin{abstract}
The deterministic model for co-infection of cervical cancer and HIV (Human Immunodeficiency Virus) diseases is formulated and rigorously analyzed. The optimal control theory is employed to the model to study the level of effort is needed to control the transmission of co-infection of cervical cancer and HIV diseases using three controls; prevention, screening and treatment control strategies. Numerical solutions show a remarkable decrease of infected individuals with HPV (Human Papilloma Virus) infection, cervical cancer, cervical cancer and HIV, cervical cancer and AIDS (Acquire Immunodeficiency Syndrome), HIV infection and AIDS after applying the combination of the optimal prevention, screening and treatment control strategies. However, Incremental Cost-Effective Ratio (ICER) shows that the best control strategy of minimizing cervical cancer among HIV-infected individuals with low cost is to use the combination of prevention and treatment control strategies.
\end{abstract}

Keywords: HPV Infection, HIV Infection, Cervical Cancer, Optimal Control, Cost-Effectiveness

\section{Introduction}

Cervical cancer is a major cause of morbidity and mortality among women in sub-Saharan countries and about $70 \%$ of cervical cancers are caused by Human Papillomavirus (HPV) types 16 and 18 which are transmitted sexually through body contact. Some studies have shown that HIV-infected women after being infected with HPV infection have a high risk to progress to HPV-related cervical diseases and invasive cervical cancer than women without having HIV infection $[1,2,3]$.

The aim of this work is to study the effect of incorporating three optimal control strategies to the co-infection model of cervical cancer and HIV diseases. In [15] formulated coinfection model of cervical cancer and HIV diseases but the findings of this paper differ from the work presented in [15] because the co-infection model incorporates three optimal control strategies; prevention, screening, and treatment.

\section{Optimal Control Analysis}

Here, we introduce optimal control strategies to the coinfection model of cervical cancer and HIV disease as presented in [15]. The co-infection model in [15] is developed as follows:

The total population of individuals at any time $t$, denoted as $N$ is categorized into ten compartments according to the individual's status of infection as follows: Susceptible individuals $(S)$, HIV-infected individuals no HPV infection $\left(I_{h}\right)$, AIDS individuals no HPV infection $\left(D_{h l}\right)$, Unscreened HPV infected individuals no HIV infection $\left(I_{p u}\right)$, Screened 
infectious individuals showing the impact of HPV infection without HIV infection $\left(I_{p s}\right)$, Individuals with cervical cancer no HIV infection, Unscreened HPV infected individuals with HIV infection $\left(I_{\text {hpu }}\right)$, Screened infectious individuals showing the impact of HPV infection with HIV infection $\left(I_{h p s}\right)$, HIV-infected individuals with cervical cancer $\left(I_{h c}\right)$ and AIDS individuals with cervical cancer $\left(D_{h l c}\right)$. Thus,

$$
N(t)=S(t)+I_{h}(t)+I_{h p u}(t)+I_{h p s}(t)+I_{p u}(t)+I_{p s}(t)+I_{h c}(t)+I_{c}(t)+D_{h l}(t)+D_{h l c}(t)
$$

The rates of transferring between different compartments are as described in Table 1.

Table 1. Description of parameters and their values for the model of co-infection HIV and cervical cancer diseases.

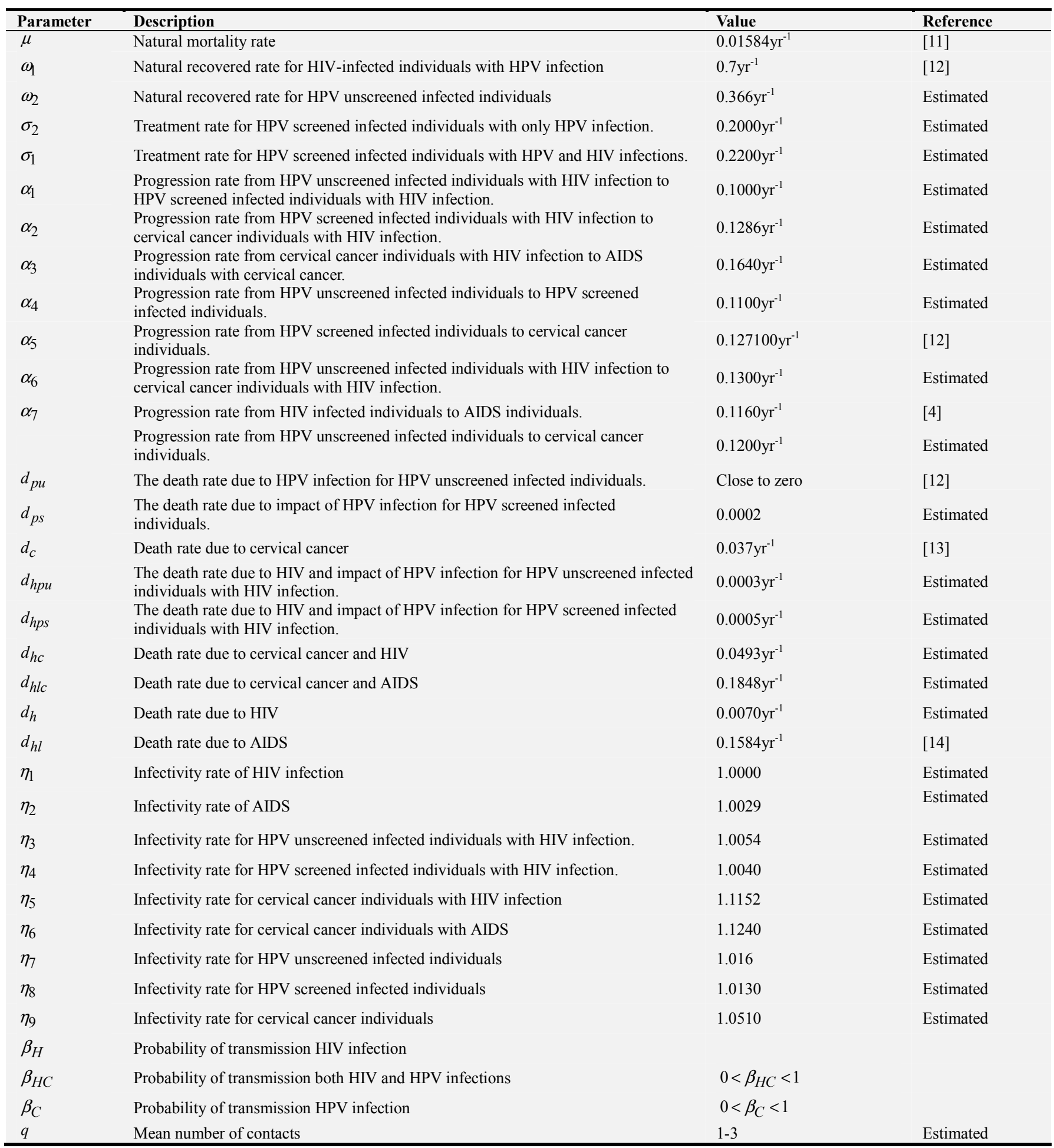


The mathematical model is defined by the following system of ordinary differential equations:

$$
\begin{aligned}
& \frac{d S}{d t}=\pi+\omega_{2} I_{p u}+\sigma_{2} I_{p s}-\left(\mu+\lambda_{H}+\lambda_{H C}+\lambda_{C}\right) S \\
& \frac{d I_{h}}{d t}=\lambda_{H} S+\omega_{1} I_{h p u}+\sigma_{1} I_{h p s}-\left(\mu+d_{h}+\alpha_{7}+\lambda_{C}\right) I_{h} \\
& \frac{d I_{h p u}}{d t}=\lambda_{H C} S+\lambda_{C} I_{h}+\lambda_{H} I_{p u}-\left(\alpha_{1}+\alpha_{6}+\omega_{1}+\mu+d_{h p u}\right) I_{h p u} \\
& \frac{d I_{p u}}{d t}=\lambda_{C} S-\lambda_{H} I_{p u}-\left(\omega_{2}+\mu+d_{p u}+\alpha_{4}+\alpha_{8}\right) I_{p u} \\
& \frac{d I_{p s}}{d t}=\alpha_{4} I_{p u}-\left(\lambda_{H}+\sigma_{2}+\alpha_{5}+\mu+d_{p s}\right) I_{p s}(1) \\
& \frac{d I_{h p s}}{d t}=\alpha_{1} I_{h p u}+\lambda_{H} I_{p s}-\left(\mu+d_{h p s}+\alpha_{2}+\sigma_{1}\right) I_{h p s} \\
& \frac{d I_{h c}}{d t}=\alpha_{2} I_{h p s}+\alpha_{6} I_{h p u}-\left(\mu+d_{h c}+\alpha_{3}\right) I_{h c} \\
& \frac{d I_{c}}{d t}=\alpha_{8} I_{p u}+\alpha_{5} I_{p s}-\left(\mu+d_{c}\right) I_{c} \\
& \frac{d D_{h l}}{d t}=\alpha_{7} I_{h}-\left(\mu+d_{h l}\right) D_{h l} \\
& \frac{d D_{h l c}}{d t}=\alpha_{3} I_{h c}-\left(\mu+d_{h l c}\right) D_{h l c}
\end{aligned}
$$

where

$$
\begin{gathered}
\lambda_{H}=\frac{\beta_{H} q}{N}\left(\eta_{1} I_{h}+\eta_{2} D_{h l}\right) \text { with } \eta_{2}>\eta_{1} \\
\lambda_{H C}=\frac{\beta_{H C} q}{N}\left(\eta_{3} I_{h p u}+\eta_{4} I_{h p s}+\eta_{5} I_{h c}+\eta_{6} D_{h l c}\right) \text { with } \eta_{6}>\eta_{5}>\eta_{3}>\eta_{4} \\
\lambda_{C}=\frac{\beta_{C} q}{N}\left(\eta_{7} I_{p u}+\eta_{8} I_{p s}+\eta_{9} I_{c}\right) \text { with } \eta_{9}>\eta_{7}>\eta_{8} .
\end{gathered}
$$

Hence, the system (1) is modified by introducing time-dependent control; $u_{1}(t)$ represents prevention control strategy (Education campaign and health hygiene practice), $u_{2}(t)$ represents the treatment of early stages of cervical cancer and $u_{3}(t)$ represents screening individuals showing an impact of HPV infection leads to cervical cancer. The following system of equations is obtained

$$
\begin{gathered}
\frac{d S}{d t}=\pi+\left(u_{2}+\sigma_{2}\right) I_{p s}+\omega_{2} I_{p u}-\left(1-u_{1}\right)\left(\lambda_{H}+\lambda_{H C}+\lambda_{C}\right) S-\mu S \\
\frac{d I_{h}}{d t}=\left(1-u_{1}\right)\left(\lambda_{H} S-\lambda_{C} I_{h}\right)+\left(u_{2}+\sigma_{1}\right) I_{h p s}+\omega_{1} I_{h p u}-\left(\alpha_{7}+d_{h}+\mu\right) I_{h} \\
\frac{d I_{h p u}}{d t}=\left(1-u_{1}\right)\left(\lambda_{H C} S+\lambda_{H} I_{p u}+\lambda_{C} I_{h}\right)-\left(u_{3}+\alpha_{1}\right) I_{h p u}-\left(\alpha_{6}+d_{h p u}+\omega_{1}+\mu\right) I_{h p u}
\end{gathered}
$$




$$
\begin{gathered}
\frac{d I_{p u}}{d t}=\left(1-u_{1}\right)\left(\lambda_{C} S-\lambda_{H} I_{h p u}\right)-\left(u_{3}+\alpha_{4}\right) I_{h p u}-\left(\alpha_{8}+d_{p u}+\omega_{2}+\mu\right) I_{h p u} \\
\frac{d I_{h p s}}{d t}=\left(u_{3}+\alpha_{1}\right) I_{h p u}+\left(1-u_{1}\right) \lambda_{H} I_{p s}-\left(u_{2}+\sigma_{1}\right) I_{h p s}-\left(\alpha_{2}+d_{h p s}+\mu\right) I_{h p s} \\
\frac{d I_{p s}}{d t}=\left(u_{3}+\alpha_{4}\right) I_{p u}-\left(1-u_{1}\right) \lambda_{H} I_{p s}-\left(u_{2}+\sigma_{2}\right) I_{p s}-\left(\alpha_{5}+d_{p s}+\mu\right) I_{p s} \\
\frac{d I_{h c}}{d t}=\alpha_{2} I_{h p s}+\alpha_{6} I_{h p u}-\left(\mu+d_{h c}+\alpha_{3}\right) I_{h c} \\
\frac{d I_{c}}{d t}=\alpha_{5} I_{p s}+\alpha_{8} I_{p u}-\left(\mu+d_{c}\right) I_{c} \\
\frac{d D_{h l}}{d t}=\alpha_{7} I_{h}-\left(\mu+d_{h l}\right) D_{h l} \\
\frac{d D_{h l c}}{d t}=\alpha_{3} I_{h c}-\left(\mu+d_{h l c}\right) D_{h l c}
\end{gathered}
$$

The objective functional is defined as follows

$$
J\left(u_{1}, u_{2}, u_{3}\right)=\lim _{u_{1}, u_{2}, u_{3}} \int_{0}^{t_{f}}\left(A_{1} I_{h p u}+A_{2} I_{p u}+A_{3} I_{h p s}+A_{4} I_{p s}+B_{1} \frac{u_{1}^{2}}{2}+B_{2} \frac{u_{2}^{2}}{2}+B_{3} \frac{u_{3}^{2}}{2}\right) d t
$$

where $A_{i}$ and $B_{j}$ for $i=1,2,3,4$ and $j=1,2,3$ are positive weights. The term $\frac{B_{1} u_{1}^{2}}{2}$ represents the cost of control effort on prevention strategy against HPV infection, $\frac{B_{2} u_{2}^{2}}{2}$ represents the cost of control effort on screening individuals with or without HIV infection having HPV infection and $\frac{B_{3} u_{3}^{2}}{2}$ represents the cost of control effort on treating individuals having cervical cancer with or without HIV infection. The main goal of introducing time-dependent controls in the co-infection model is to prevent women not to acquire HPV infection which leads to cervical cancer and to minimize infected women showing the impact of HPV infection lead to cervical cancer while minimizing the cost of controls $u_{1}(t), u_{2}(t)$ and $u_{3}(t)$ as in $[5,6,7]$. The goal is to seek an optimal control $u_{1}^{*}, u_{2}^{*}$ and $u_{3}^{*}$ numerically such that

$$
J\left(u_{1}^{*}, u_{2}^{*}, u_{3}^{*}\right)=\min \left\{J\left(u_{1}, u_{2}, u_{3}\right) \mid u_{1}, u_{2}, u_{3} \in \Omega\right\}
$$

where $\Omega=\left\{\left(u_{1}, u_{2}, u_{3}\right) \quad\right.$ such that $u_{1}, u_{2}, u_{3} \quad$ measurable with $0 \leq u_{1} \leq 1,0 \leq u_{2} \leq 1$ and $0 \leq u_{1} \leq 1$ for $\left.t \in\left[0, t_{f}\right]\right\}$ is the control set. The necessary conditions that an optimal must satisfy come from the Pontryagin's maximum principle [8]. This principle converts (2) and (3) into theproblem of minimizing point-wise Hamiltonian, $H$, with respect to $u_{1}$, $u_{2}$ and $u_{3}$.

$$
\begin{aligned}
H & =A_{1} I_{h p u}+A_{2} I_{p u}+A_{3} I_{h p s}+A_{4} I_{p s}+B_{1} \frac{u_{1}^{2}}{2}+B_{2} \frac{u_{2}^{2}}{2}+B_{3} \frac{u_{3}^{2}}{2} \\
& +M_{S}\left(\pi+\left(u_{2}+\sigma_{2}\right) I_{p s}+\omega_{2} I_{p u}-\left(1-u_{1}\right)\left(\lambda_{H}+\lambda_{H C}+\lambda_{C}\right) S-\mu S\right) \\
& +M_{I_{h}}\left(\left(1-u_{1}\right)\left(\lambda_{H} S-\lambda_{C} I_{h}\right)+\left(u_{2}+\sigma_{1}\right) I_{h p s}+\omega_{1} I_{h p u}-\left(\alpha_{7}+d_{h}+\mu\right) I_{h}\right) \\
& +M_{I_{h p u}}\left(\left(1-u_{1}\right)\left(\lambda_{H C} S+\lambda_{H} I_{p u}+\lambda_{C} I_{h}\right)-\left(u_{3}+\alpha_{1}\right) I_{h p u}-\left(\alpha_{6}+d_{h p u}+\omega_{1}+\mu\right) I_{h p u}\right) \\
& +M_{I_{p u}}\left(\left(1-u_{1}\right)\left(\lambda_{C} S-\lambda_{H} I_{h p u}\right)-\left(u_{3}+\alpha_{4}\right) I_{h p u}-\left(\alpha_{8}+d_{p u}+\omega_{2}+\mu\right) I_{h p u}\right)
\end{aligned}
$$




$$
\begin{aligned}
& +M_{I_{h p s}}\left(\left(u_{3}+\alpha_{1}\right) I_{h p u}+\left(1-u_{1}\right) \lambda_{H} I_{p s}-\left(u_{2}+\sigma_{1}\right) I_{h p s}-\left(\alpha_{2}+d_{h p s}+\mu\right) I_{h p s}\right) \\
& +M_{I_{p s}}\left(\left(u_{3}+\alpha_{4}\right) I_{p u}-\left(1-u_{1}\right) \lambda_{H} I_{p s}-\left(u_{2}+\sigma_{2}\right) I_{p s}-\left(\alpha_{5}+d_{p s}+\mu\right) I_{p s}\right) \\
& +M_{I_{h c}}\left(\alpha_{2} I_{h p s}+\alpha_{6} I_{h p u}-\left(\mu+d_{c h}+\alpha_{3}\right) I_{h c}\right) \\
& +M_{I_{c}}\left(\alpha_{5} I_{p s}+\alpha_{8} I_{p u}-\left(\mu+d_{c}\right) I_{c}\right) \\
& +M_{D_{h l}}\left(\alpha_{7} I_{h}-\left(\mu+d_{h l}\right) D_{h l}\right) \\
& \quad+M_{D_{h l c}}\left(\alpha_{3} I_{h c}-\left(\mu+d_{h l c}\right) D_{h l c}\right)
\end{aligned}
$$

where, $M_{S}, M_{I_{h}}, M_{I_{h p u}}, M_{I_{p u}}, M_{I_{h p s}}, M_{I_{p s}}, M_{I_{h c}}, M_{I_{c}}, M_{D_{h l}}$ and $M_{D_{h l c}}$ are the co-state variables or the adjoint variables.

Theorem: For the optimal control triples $u_{1}^{*}, u_{2}^{*}$ and $u_{3}^{*}$ that minimize $J\left(u_{1}, u_{2}, u_{3}\right)$ over $\Omega$, there exist adjoint variables $M_{S}, M_{I_{h}}, M_{I_{h p u}}, M_{I_{p u}}, M_{I_{h p s}}$,

$$
M_{I_{p s}}, M_{I_{h c}}, M_{I_{c}}, M_{D_{h l}} \text { and } M_{D_{h l c}} \text { satisfying }
$$

$$
\frac{-d M_{j}}{d t}=\frac{\partial H}{\partial j}
$$

where $j=S, I_{h}, I_{h p u}, I_{p u}, I_{h p s}, I_{h c}, I_{c}, D_{h l}, D_{h l c}$ and with transversality condition $M_{S}(t)=M_{I_{h}}(t)=M_{I_{h p u}}(t)=M_{I_{p u}}(t)=M_{I_{h p s}}(t)=M_{I_{p s}}(t)=M_{I_{h c}}(t)=M_{I_{c}}(t)=M_{D_{h l}}\left(t_{f}\right)=M_{D_{h l c}}\left(t_{f}\right)=0$

and,

$$
\begin{aligned}
& u_{1}^{*}=\max \left\{0, \min \left(\begin{array}{l}
1, \\
\left.\frac{1}{B_{1}}\left(\begin{array}{l}
I_{p s} \lambda_{H}\left(M_{I_{h p s}}-M_{I_{p s}}\right)+S \lambda_{H}\left(M_{I_{H}}-M_{S}\right)+I_{p u} \lambda_{H}\left(M_{I_{h p u}}-M_{I_{p u}}\right) \\
+S \lambda_{H C}\left(M_{I_{h p u}}-M_{S}\right)+S \lambda_{C}\left(M_{I_{p u}}-M_{S}\right)+\lambda_{C} I_{H}\left(M_{I_{h p u}}-M_{I_{h}}\right)
\end{array}\right)\right)
\end{array}\right)\right. \\
& u_{2}^{*}=\max \left\{0, \min \left(1, \frac{1}{B_{2}}\left(I_{h p s}\left(M_{I_{h p s}}-M_{I_{h}}\right)+I_{p s}\left(M_{I_{p s}}-M_{S}\right)\right)\right)\right\} \\
& u_{3}^{*}=\max \left\{0, \min \left(1, \frac{1}{B_{3}}\left(I_{h p u}\left(M_{I_{h p u}}-M_{I_{h p s}}\right)+I_{p u}\left(M_{I_{p u}}-M_{I_{p s}}\right)\right)\right)\right\}
\end{aligned}
$$

Proof. Corollary 4.1 of Freming and Riches [9] gives the existence of an optimal control due to the convexity of the integrand $J$ with respect to $u_{1}, u_{2}$ and $u_{3}$ a priori boundedness of the state solutions and the Lipchitz property of the state system with respect to the state variables. Differentiating Hamiltonian functions with respect to state variables gives differential equations governing the adjoint variables as follows;

$$
\begin{gathered}
-\frac{d M_{S}}{d t}=M_{S} \mu+\frac{I_{p s} \lambda_{H}\left(1-u_{1}\right)\left(M_{I_{h p s}}-M_{I_{p s}}\right)}{N}+\frac{I_{p u} \lambda_{H}\left(1-u_{1}\right)\left(M_{I_{h p u}}-M_{I_{p u}}\right)}{N} \\
+\lambda_{H C}\left(1-u_{1}\right)\left(M_{S}-M_{I_{h p u}}\right)+\frac{I_{h} \lambda_{C}\left(1-u_{1}\right)\left(M_{I_{h p u}}-M_{I_{h}}\right)}{N}+\lambda_{H}\left(1-u_{1}\right)\left(M_{S}-M_{I_{h}}\right)
\end{gathered}
$$




$$
\begin{aligned}
& +\lambda_{C}\left(1-u_{1}\right)\left(M_{S}-M_{I_{h p s}}\right)+\frac{S\left(1-u_{1}\right) \lambda_{H}\left(M_{I_{h}}-M_{S}\right)}{N}+\frac{S\left(1-u_{1}\right) \lambda_{H C}\left(M_{I_{\text {hpu }}}-M_{S}\right)}{N} . \\
& -\frac{d M_{I_{h}}}{d t}=\alpha_{7} M_{D_{h l}}+M_{I_{h}}\left(\mu+d_{h}\right)+\frac{I_{p s} q\left(1-u_{1}\right) \beta_{H} \eta_{1}\left(M_{I_{p s}}-M_{I_{h p s}}\right)}{N}+\frac{I_{p s} \lambda_{H}\left(1-u_{1}\right)\left(M_{I_{h p s}}-M_{I_{p s}}\right)}{N} \\
& +\frac{S \beta_{H} q \eta_{1}\left(1-u_{1}\right)\left(M_{S}-M_{I_{p s}}\right)}{N}+\frac{I_{p u} \beta_{H} q \eta_{1}\left(1-u_{1}\right)\left(M_{I_{p u}}-M_{I_{h p u}}\right)}{N}+\frac{S\left(1-u_{1}\right) \lambda_{H C}\left(M_{I_{h p u}}-M_{S}\right)}{N} \\
& +\frac{I_{p u} \lambda_{H}\left(1-u_{1}\right)\left(M_{I_{h p u}}-M_{I_{h p s}}\right)}{N}+\frac{I_{h} \lambda_{C}\left(1-u_{1}\right)\left(M_{I_{h p u}}-M_{I_{h}}\right)}{N}+\frac{S \lambda_{H}\left(1-u_{1}\right)\left(M_{I_{h}}-M_{S}\right)}{N} \\
& +\lambda_{C}\left(1-u_{1}\right)\left(M_{I_{h}}-M_{I_{\text {hpu }}}\right)+\frac{S \lambda_{C}\left(1-u_{1}\right)\left(M_{I_{p u}}-M_{S}\right)}{N} \\
& -\frac{d M_{I_{h p u}}}{d t}=-A_{1}+\alpha_{6}\left(M_{I_{h p u}}-M_{I_{h c}}\right)+M_{I_{h p u}}\left(\mu+d_{h p u}\right)+\left(u_{3}+\alpha_{1}\right)\left(M_{I_{h p u}}-M_{I_{h p s}}\right)+\omega_{1}\left(M_{I_{h p u}}-M_{I_{h}}\right) \\
& +\frac{I_{p s}\left(1-u_{1}\right) \lambda_{H}\left(M_{I_{h p s}}-M_{I_{p s}}\right)}{N}+\frac{S \lambda_{H}\left(1-u_{1}\right)\left(M_{I_{h}}-M_{S}\right)}{N}+\frac{S q\left(1-u_{1}\right) \beta_{H C} \eta_{3}\left(M_{S}-M_{I_{h p u}}\right)}{N} \\
& +\frac{I_{p u}\left(1-u_{1}\right) \lambda_{H}\left(M_{I_{h p u}}-M_{I_{p u}}\right)}{N}+\frac{\lambda_{C} I_{h}\left(1-u_{1}\right)\left(M_{I_{h p u}}-M_{I_{h}}\right)}{N}+\frac{S \lambda_{H C}\left(1-u_{1}\right)\left(M_{I_{h p u}}-M_{S}\right)}{N} \\
& +\frac{S \lambda_{C}\left(1-u_{1}\right)\left(M_{I_{p u}}-M_{S}\right)}{N} . \\
& -\frac{d M_{I_{p u}}}{d t}=-A_{2}+\alpha_{8}\left(M_{I_{p u}}-M_{I_{c}}\right)+\frac{I_{p s} \lambda_{H}\left(1-u_{1}\right)\left(M_{I_{h p s}}-M_{I_{p s}}\right)}{N}+\frac{I_{p u} \lambda_{H}\left(1-u_{1}\right)\left(M_{I_{h p u}}-M_{I_{p u}}\right)}{N} \\
& +\frac{I_{h}\left(1-u_{1}\right) q \beta_{C} \eta_{7}\left(M_{I_{h}}-M_{I_{\text {hpu }}}\right)}{N}+M_{I_{p u}}\left(\mu+d_{p u}\right)+\lambda_{H}\left(1-u_{1}\right)\left(M_{I_{p u}}-M_{I_{h p u}}\right)+\omega_{2}\left(M_{I_{p u}}-M_{S}\right) \\
& +\frac{I_{h} \lambda_{C}\left(1-u_{1}\right)\left(M_{I_{h p u}}-M_{I_{h}}\right)}{N}+\left(u_{3}+\alpha_{4}\right)\left(M_{I_{p u}}-M_{I_{p s}}\right)+\frac{S\left(1-u_{1}\right) \lambda_{H C}\left(M_{I_{h p u}}-M_{S}\right)}{N} \\
& +\frac{S\left(1-u_{1}\right) \lambda_{C}\left(M_{I_{p u}}-M_{S}\right)}{N}+\frac{S\left(1-u_{1}\right) \lambda_{H}\left(M_{I_{h}}-M_{S}\right)}{N}+\frac{S q\left(1-u_{1}\right) \beta_{C} \eta_{7}\left(M_{S}-M_{I_{p u}}\right)}{N} \\
& -\frac{d M_{I_{h p s}}}{d t}=-A_{3}+\alpha_{2}\left(M_{I_{h p s}}-M_{I_{h c}}\right)+\frac{I_{p s}\left(1-u_{1}\right) \lambda_{H}\left(M_{I_{p s}}-M_{I_{h}}\right)}{N}+\frac{S \lambda_{h}\left(1-u_{1}\right)\left(M_{I_{h}}-M_{S}\right)}{N} \\
& +\frac{I_{h}\left(1-u_{1}\right) \lambda_{C}\left(M_{I_{h p u}}-M_{I_{h}}\right)}{N}+\left(u_{3}+\sigma_{1}\right)\left(M_{I_{h p s}}-M_{I_{h}}\right)+M_{I_{h p s}}\left(\mu+d_{h p s}\right)+\frac{I_{p u} \lambda_{H}\left(1-u_{1}\right)\left(M_{I_{h p u}}-M_{I_{p u}}\right)}{N}
\end{aligned}
$$




$$
\begin{aligned}
& +\frac{S q\left(1-u_{1}\right) \beta_{H C} \eta_{4}\left(M_{S}-M_{I_{h p u}}\right)}{N}+\frac{S \lambda_{C}\left(1-u_{1}\right)\left(M_{I_{p u}}-M_{S}\right)}{N}+\frac{S\left(1-u_{1}\right) \lambda_{H C}\left(M_{I_{h p u}}-M_{S}\right)}{N} \\
& -\frac{d M_{I_{p s}}}{N}=-A_{4}+\alpha_{5}\left(M_{I_{p s}}-M_{I_{h c}}\right)+M_{I_{p s}}\left(\mu+d_{p s}\right)+\left(u_{2}+\sigma_{1}\right)\left(M_{I_{p s}}-M_{S}\right) \\
& +\frac{I_{p s} \lambda_{H}\left(1-u_{1}\right)\left(M_{I_{h p s}}-M_{I_{p s}}\right)}{N}+\left(1-u_{1}\right) \lambda_{H}\left(M_{I_{p s}}-M_{I_{h p s}}\right)+\left(1-u_{1}\right) I_{p u} \lambda_{H}\left(M_{I_{h p u}}-M_{I_{p u}}\right) \\
& +\frac{S\left(1-u_{1}\right) \beta_{C} \eta_{8} q\left(M_{S}-M_{I_{p u}}\right)}{N}+\frac{S \lambda_{C}\left(1-u_{1}\right)\left(M_{I_{p u}}-M_{S}\right)}{N}+\frac{I_{h} \lambda_{C}\left(1-u_{1}\right)\left(M_{I_{h p u}}-M_{I_{h}}\right)}{N} \\
& +\frac{I_{h}\left(1-u_{1}\right) \beta_{C} \eta_{8} q\left(M_{I_{h}}-M_{I_{\text {hpu }}}\right)}{N}+\frac{S\left(1-u_{1}\right) \lambda_{H}\left(M_{I_{h}}-M_{S}\right)}{N}+\frac{S\left(1-u_{1}\right) \lambda_{H C}\left(M_{I_{\text {hpu }}}-M_{S}\right)}{N} \\
& -\frac{d M_{I_{h c}}}{d t}=M_{I_{h c}}\left(\mu+d_{c h}\right)+\alpha_{3}\left(M_{I_{h c}}-M_{D_{h l c}}\right)+\frac{I_{p s} \lambda_{H}\left(1-u_{1}\right)\left(M_{I_{h p s}}-M_{I_{p s}}\right)}{N}+\frac{S \lambda_{H}\left(1-u_{1}\right)\left(M_{I_{h}}-M_{S}\right)}{N} \\
& +\frac{S q \beta_{H C} \eta_{5}\left(M_{S}-M_{I_{\text {hpu }}}\right)}{N}+\frac{S \lambda_{H C}\left(1-u_{1}\right)\left(M_{I_{\text {hpu }}}-M_{S}\right)}{N}+\frac{S \lambda_{C}\left(1-u_{1}\right)\left(M_{I_{\text {hpu }}}-M_{S}\right)}{N} \\
& +\frac{I_{p u} \lambda_{H}\left(1-u_{1}\right)\left(M_{I_{h p u}}-M_{I_{p u}}\right)}{N}+\frac{I_{h} \lambda_{C}\left(1-u_{1}\right)\left(M_{I_{h p u}}-M_{I_{h}}\right)}{N} \\
& -\frac{d M_{I_{c}}}{d t}=M_{I_{c}}\left(\mu+d_{c}\right)+\frac{I_{p s} \lambda_{H}\left(M_{I_{h p s}}-M_{I_{p s}}\right)}{N}+\frac{S \lambda_{H}\left(1-u_{1}\right)\left(M_{I_{h}}-M_{S}\right)}{N}+\frac{S \lambda_{H C}\left(1-u_{1}\right)\left(M_{I_{h p u}}-M_{S}\right)}{N} \\
& +\frac{S \beta_{C} q \eta_{9}\left(1-u_{1}\right)\left(M_{S}-M_{I_{p u}}\right)}{N}+\frac{I_{p u} \lambda_{H}\left(1-u_{1}\right)\left(M_{I_{h p u}}-M_{I_{p u}}\right)}{N}+\frac{S \lambda_{C}\left(1-u_{1}\right)\left(M_{I_{p u}}-M_{S}\right)}{N} \\
& +\frac{I_{h} \beta_{C} q \eta_{9}\left(1-u_{1}\right)\left(M_{I_{h}}-M_{I_{\text {hpu }}}\right)}{N}+\frac{I_{h} \lambda_{C}\left(1-u_{1}\right)\left(M_{I_{\text {hpu }}}-M_{I_{h}}\right)}{N} \\
& -\frac{d M_{D_{h l}}}{d t}=\left(\mu+d_{h l}\right) M_{D_{h l}}+\frac{I_{p s} \beta_{h} q \eta_{2}\left(1-u_{1}\right)\left(M_{I_{p s}}-M_{I_{h p s}}\right)}{N}+\frac{I_{p s}\left(1-u_{1}\right) \lambda_{H}\left(M_{I_{h p s}}-M_{I_{p s}}\right)}{N} \\
& +\frac{S \beta_{H} \eta_{2} q\left(1-u_{1}\right)\left(M_{S}-M_{I_{h}}\right)}{N}+\frac{S \lambda_{H}\left(1-u_{1}\right)\left(M_{I_{h}}-M_{S}\right)}{N}+\frac{S \lambda_{H C}\left(1-u_{1}\right)\left(M_{I_{\text {hpu }}}-M_{S}\right)}{N} \\
& +\frac{I_{h} \lambda_{C}\left(1-u_{1}\right)\left(M_{I_{h p u}}-M_{I_{h}}\right)}{N}+\frac{I_{p u} \lambda_{H}\left(1-u_{1}\right)\left(M_{I_{h p u}}-M_{I_{p u}}\right)}{N}+\frac{I_{p u} \beta_{H} \eta_{2} q\left(1-u_{1}\right)\left(M_{I_{p u}}-M_{I_{h p u}}\right)}{N}+\frac{S \lambda_{C}\left(1-u_{1}\right)\left(M_{I_{p u}}-M_{S}\right)}{N} \\
& -\frac{d M_{D_{h l c}}}{d t}=\left(\mu+d_{h l c}\right) M_{D_{h l c}}+\frac{\left(1-u_{1}\right) I_{p s} \lambda_{H}\left(M_{I_{h p s}}-M_{I_{p s}}\right)}{N}+\frac{S\left(1-u_{1}\right) \lambda_{H}\left(M_{I_{h}}-M_{S}\right)}{N}
\end{aligned}
$$


respect to each control variables as follows

$$
0=\frac{\partial H}{\partial u_{i}} \text { for } i=1,2,3
$$

Solving for $u_{1}^{*}, u_{2}^{*}$ and $u_{3}^{*}$, subject to the constraints, provides the characterization equations (7)-(9). In the next part, the numerical solutions of optimality system are discussed.

\section{Numerical Solutions}

In order to obtain optimal control solutions, the optimality system which consists of two systems namely; the state system and the adjoint system is solved. In solving state equations using forward fourth order Runge-Kutta, an initial guess of all controls over time are made and the initial value of state variables are introduced. Having the solution of state functions and the value of optimal controls, the adjoint equations are solved using backward fourth order Runge-
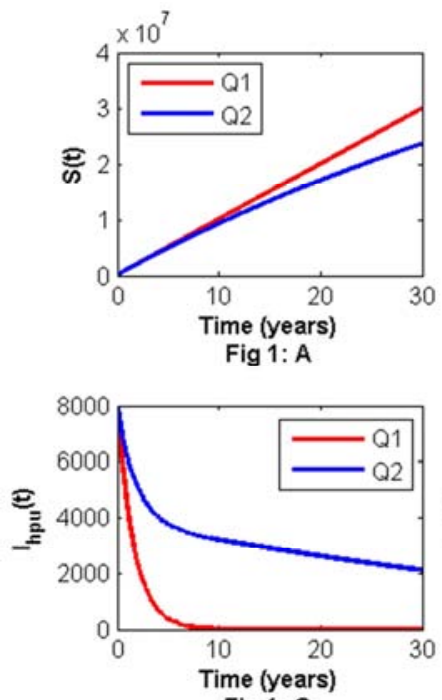

Fig 1: C

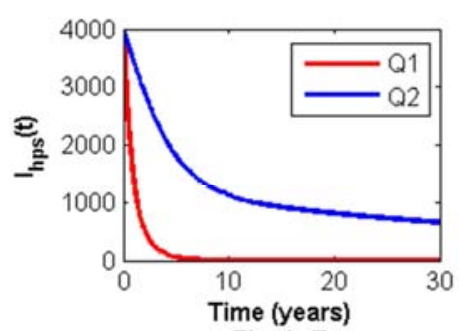

Fig 1: E

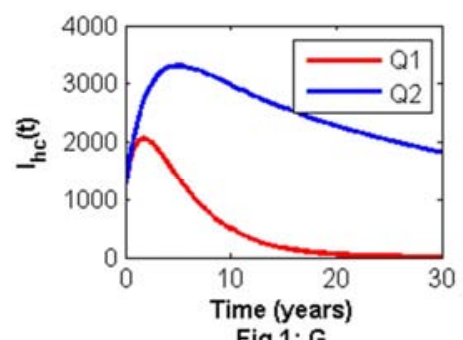

Fig 1: G
Kutta by using transversality condition. In this simulation, the weights are chosen be $A_{1}=80, A_{2}=75, A_{3}=50$, $A_{4}=45, B_{1}=100, B_{2}=110$ and $B_{3}=120$. Other parameter descriptions and values used in getting numerical results of the co-infection model of cervical cancer and HIV diseases are presented in Table 1. Control strategies are formed and studied numerically as follows

\subsection{Strategy I: Combination of Prevention and Treatment Control Strategies}

The combination of prevention control strategy $u_{1}$ and treatment control $u_{2}$ are used to optimize objective functional while setting screening control $u_{3}$ equal to zero. The results show that applying optimal prevention and treatment control strategies; the population of susceptible individuals increases whereas thepopulation of all infected compartments decreases as illustrated in Figure1: A and Figure1: B-J respectively.
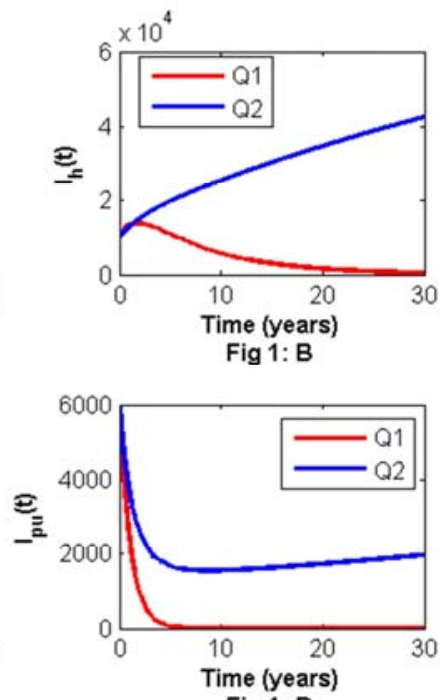

Fig 1: D

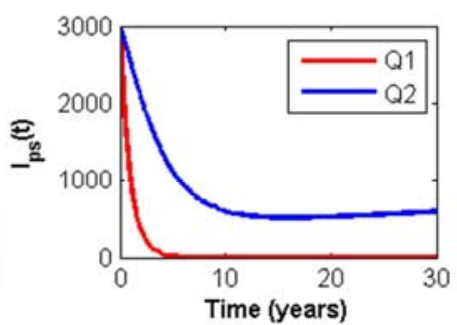

Fig 1: F

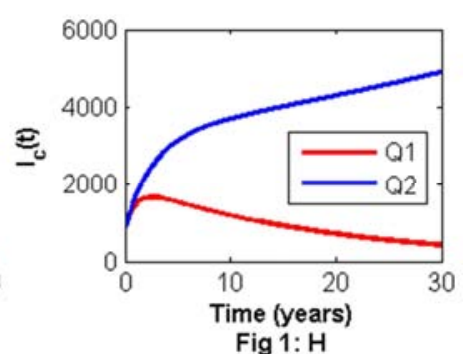

Fig 1: $\mathrm{H}$ 

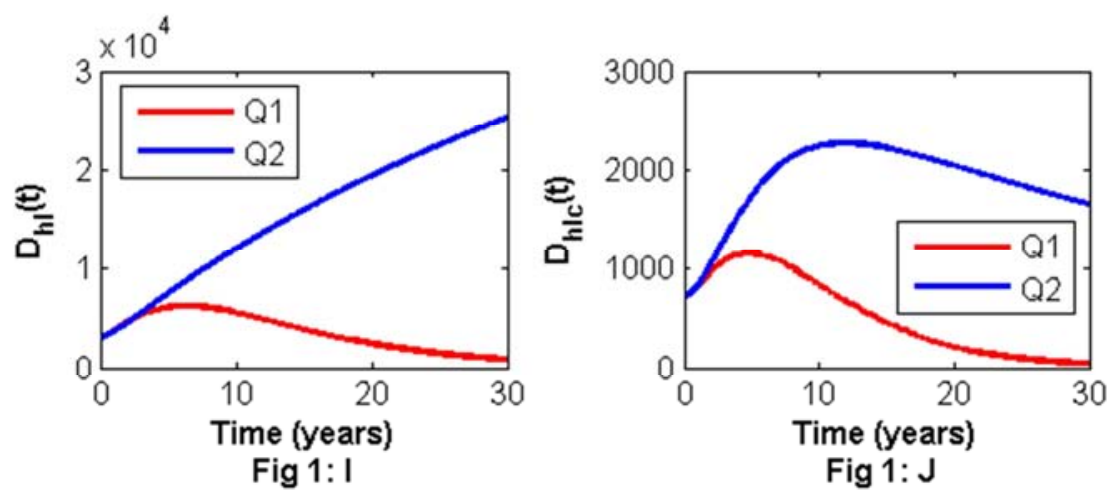

Figure 1. The use of prevention and treatment control strategies. Figure 1: A - J are time series plot of different population where $Q_{1}=u_{1} \neq 0, u_{2} \neq 0, u_{3}=0$ with control and $Q_{2}=\left(u_{1}=0, u_{2}=0, u_{3}=0\right)$ without control.

\subsection{Strategy II: Combination of Prevention Control Strategy and Screening Strategy}

The combination of prevention control strategy, $u_{1}$, and screening control strategy, $u_{3}$, are used to optimize objective

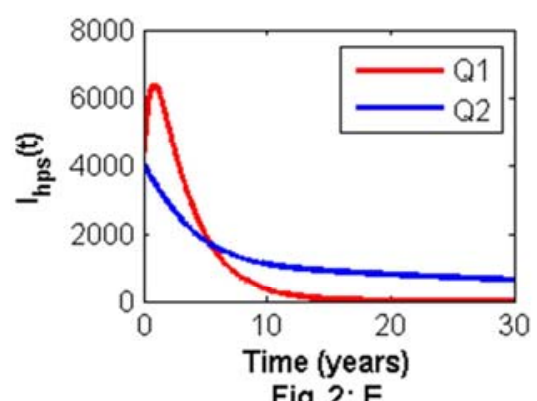

Fig 2: E

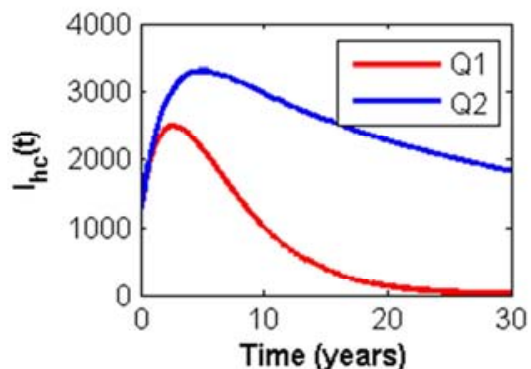

Fig 2: G

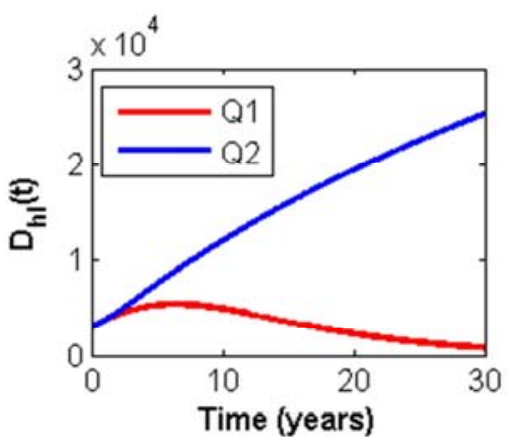

Fig 2: I functional while setting treatment control strategy, $u_{2}$, equal to zero. Results illustrate that the population of susceptible individuals increases (see Figure 2: A) while the population of infected individuals decreases (see Figure 2: B-J).
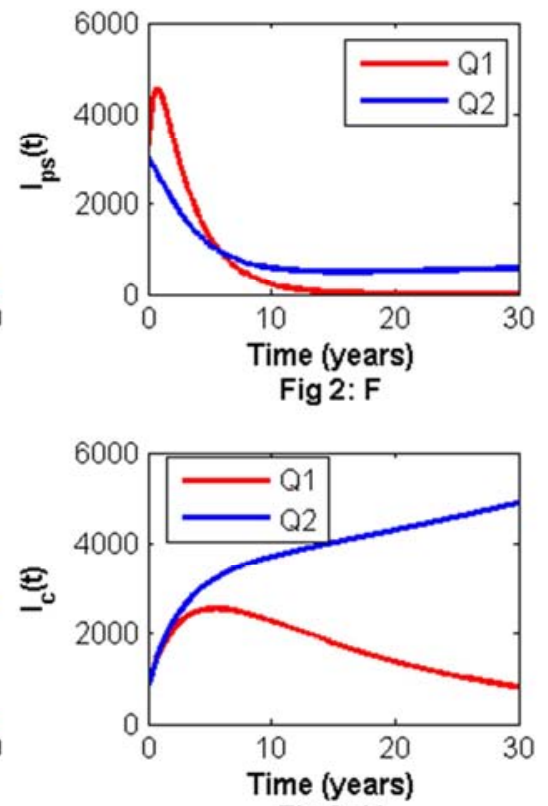

Fig 2: $\mathrm{H}$

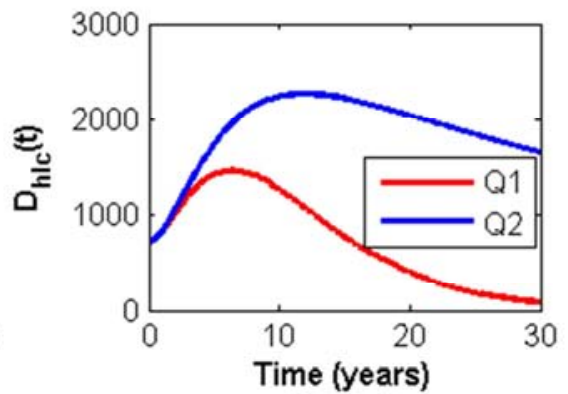

Fig 2: J 

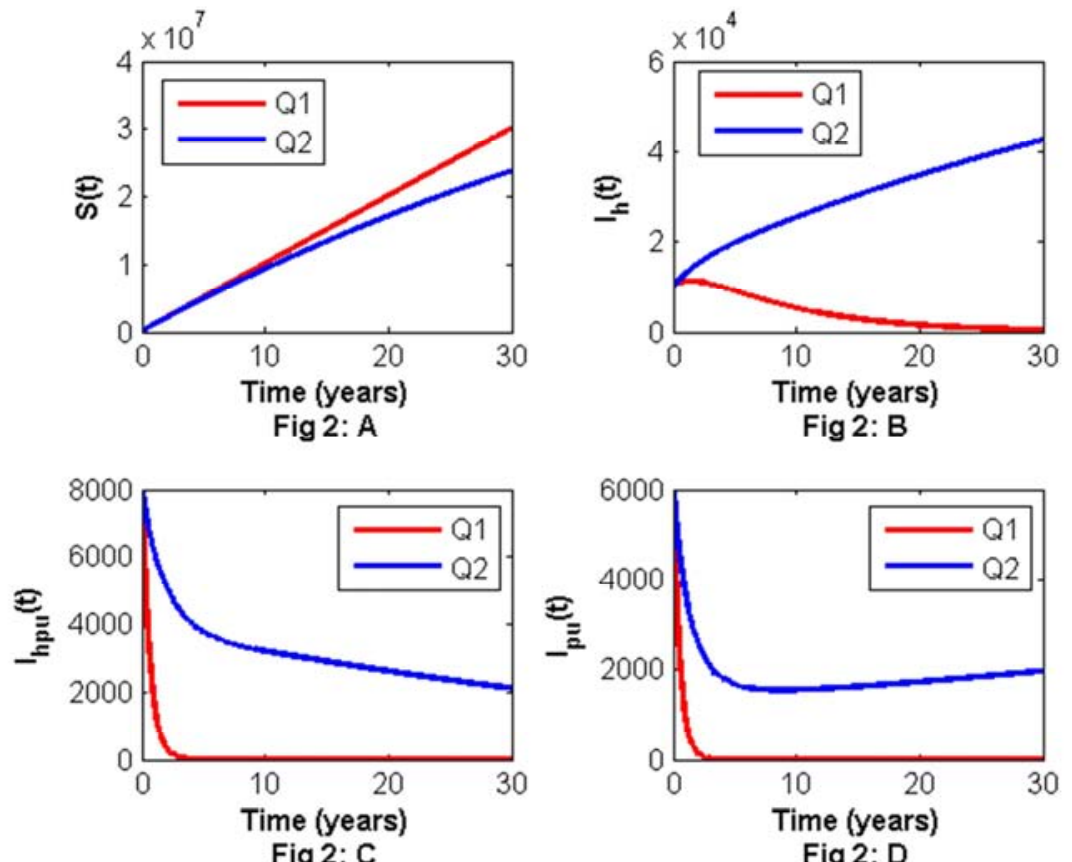

Figure 2. The useprevention and screening control strategies. Figure 2: $A-J$ are time series plot of different population where $Q_{1}=u_{1} \neq 0, u_{2}=0, u_{3} \neq 0$ with control and $Q_{2}=\left(u_{1}=0, u_{2}=0, u_{3}=0\right)$ without control.

\subsection{Strategy III: Combination of Screening and Treatment Control Strategies}

The combination of treatment control $u_{2}$ and screening control $u_{3}$ are used to optimize objective functional while setting vaccination control $u_{1}$ to zero. Results indicate that there is unremarkable increase of susceptible individuals and the population of infected individuals declines not much compared with other combination control strategies as illustrated in Figure 3: A and Figure 3: B - J respectively.
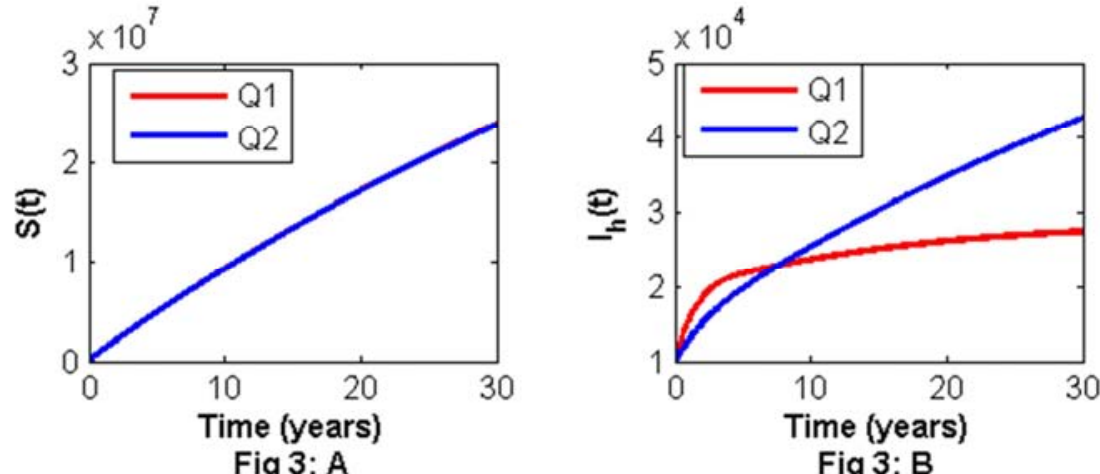

Fig 3: A

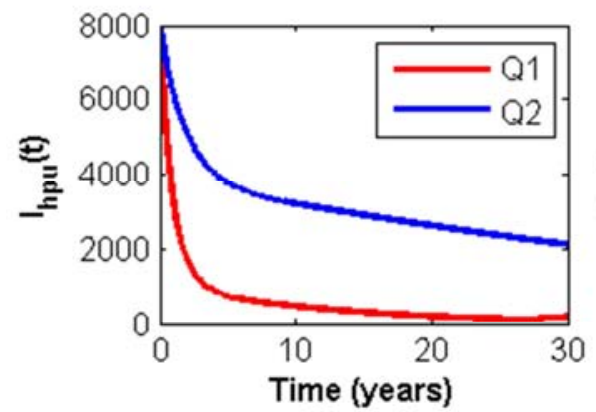

Fig 3: C

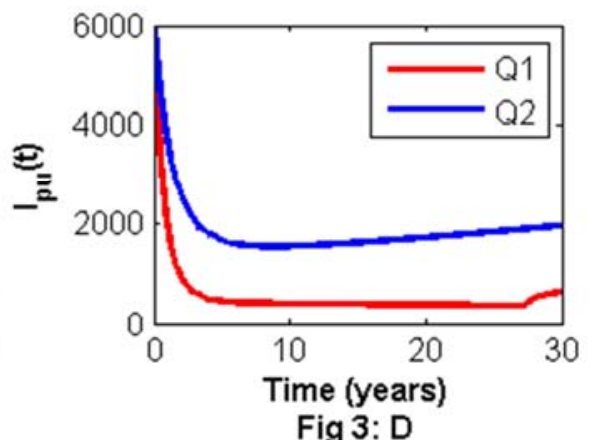



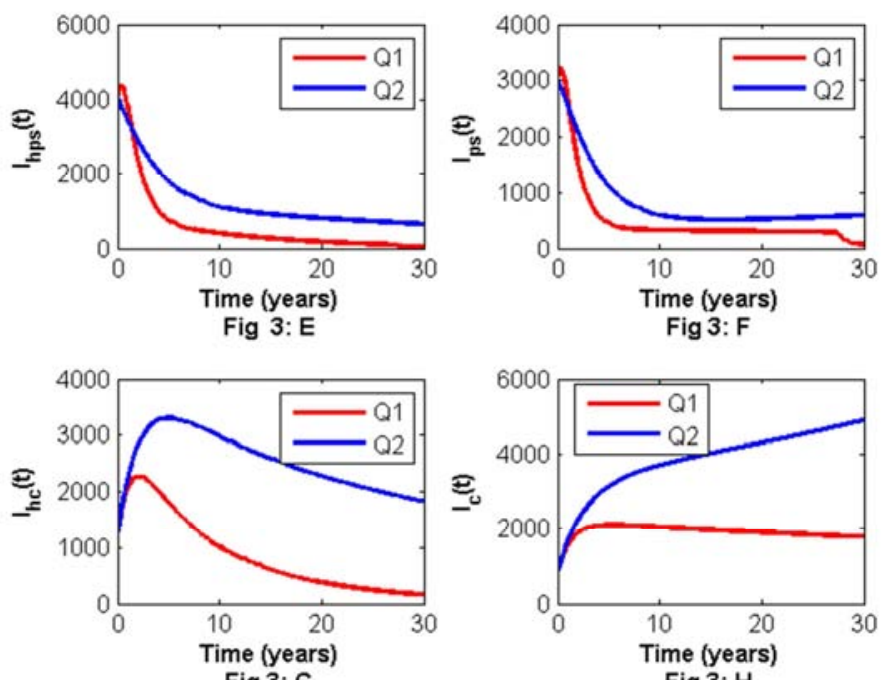

Fig 3: $\mathrm{H}$
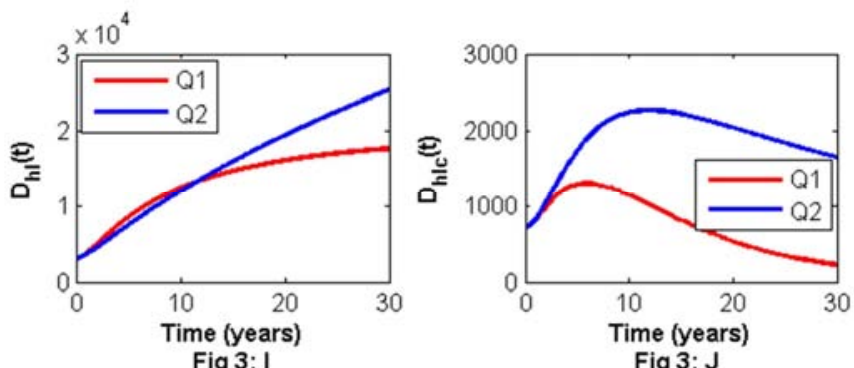

Figure 3. The use of screening and treatment control strategies. Figure 3: $A$ - J are time series plot of different population where $Q_{1}=u_{1}=0, u_{2} \neq 0, u_{3} \neq 0$ with control and $Q_{2}=\left(u_{1}=0, u_{2}=0, u_{3}=0\right)$ without control.

\subsection{Strategy IV: Combination of Prevention, Treatment and Screening Control Strategies}

The combination of prevention control strategy, treatment control strategy $u_{2}$, and screening control strategy $u_{3}$ are used to optimize objective functional. Results illustrates that
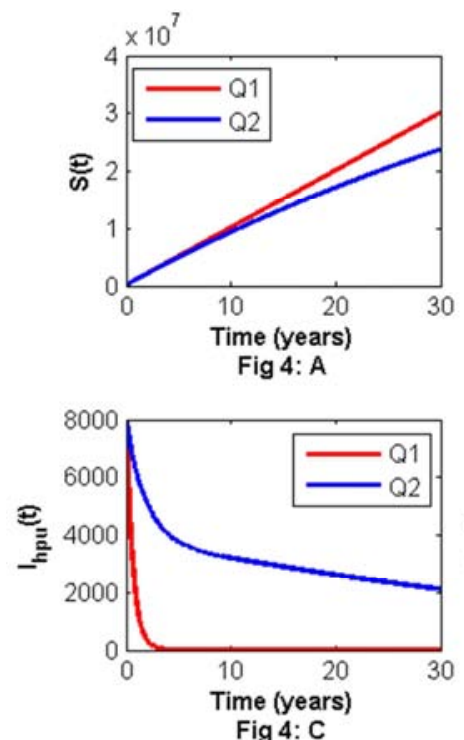

Fig 4: C the population of susceptible individuals increases as shown in Figure 4: A while the population of infected individuals decrease compared with other combination of control strategies as shown in Figure 4: B - J.
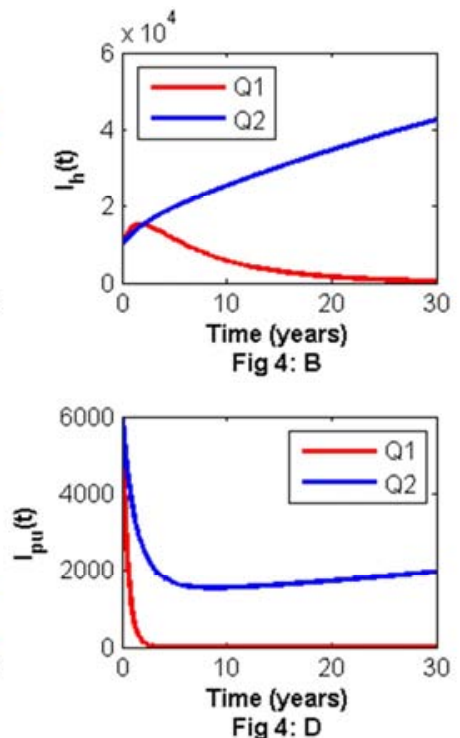

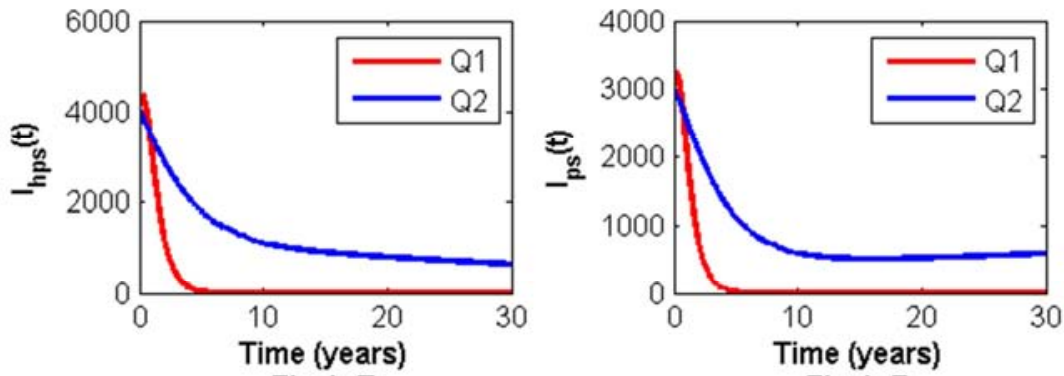

Fig 4: $E$

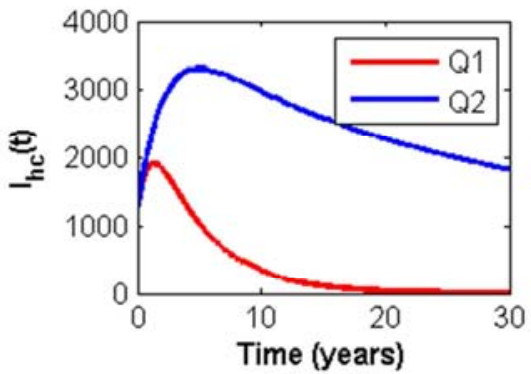

Fig 4: G

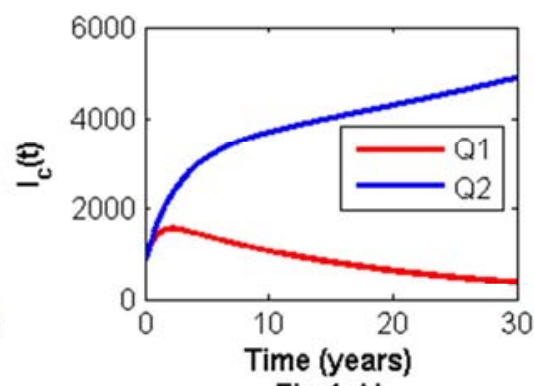

Fig 4: $\mathrm{H}$
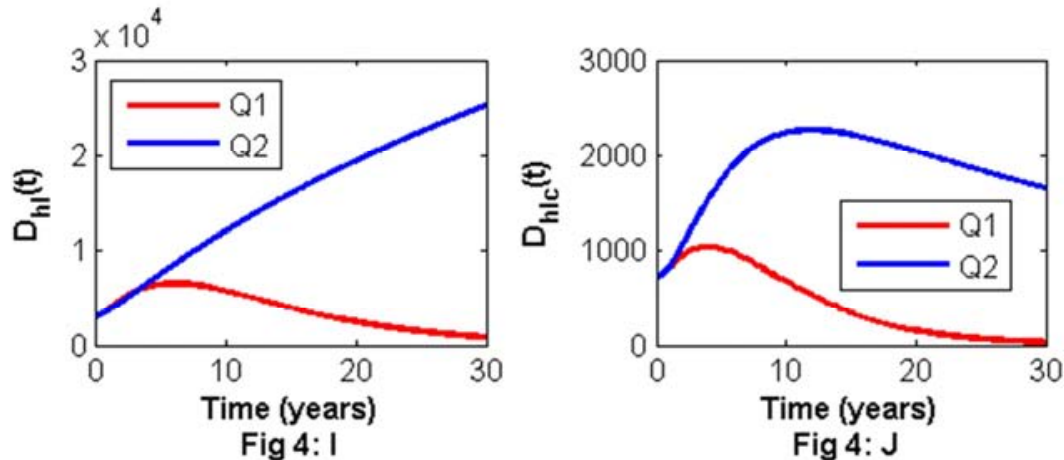

Figure 4. Showing impact of using all control strategies. Figure 4: A - J are time series plot of different population where $Q_{1}=u_{1} \neq 0, u_{2} \neq 0, u_{3} \neq 0$ with control and $Q_{2}=\left(u_{1}=0, u_{2}=0, u_{3}=0\right)$ without control.

\section{Cost-effectiveness Analysis}

Here, Incremental Cost Effectiveness Ratio (ICER) is used to quantify the cost-effectiveness of different strategies. This approach is useful to understand which strategy saves a lot of averted species while spending low cost. This technique is needed to compare more than one competing interventions strategies incrementally, one intervention should be compared with the next less effective alternative [10]. The ICER formula is given by

$\mathrm{ICER}=$

Difference in intervention cost

Difference in the total number of infection averted

The total number of infection averted is obtained by calculating the difference between the total number of new cases of individuals having HPV infection without control and the total number of new cases of individuals having HPV infection with control.
Table 2. Calculation of ICER after arranging the number of total infections averted in ascending order.

\begin{tabular}{llll}
\hline Strategy & Total Cost $\mathbf{( \$ )}$ & $\begin{array}{l}\text { Total infections } \\
\text { Averted }\end{array}$ & ICER \\
\hline Strategy III & $\$ 2,480.00$ & $64,637.00$ & 0.0384 \\
Strategy II & $\$ 2,770.20$ & $75,510.00$ & 0.0266 \\
Strategy I & $\$ 3,090.00$ & $91,109.00$ & 0.0205 \\
Strategy IV & $\$ 4,383.30$ & $93,870.00$ & 0.4684 \\
\hline
\end{tabular}

$$
\begin{aligned}
& \operatorname{ICER}(\text { Strategy III })=\frac{2,480.50}{64,637.00}=0.0384, \\
& \operatorname{ICER}(\text { Strategy II })=\frac{2,770.20-2,480.50}{75,510.00-64,637.00}=0.0266, \\
& \operatorname{ICER}(\text { Strategy I })=\frac{3,090.00-2,770.20}{91,109.00-75,510.00}=0.0205 \text { and } \\
& \operatorname{ICER}(\text { Strategy IV })=\frac{4,383.30-3,090.00}{93,870.00-91,109.00}=0.4684 .
\end{aligned}
$$

Comparing strategy III and strategy II, ICER of strategy II 
is less than ICER of strategy III. Hence strategy III is more costly and less effective than strategy II. Thus, strategy III is omitted and ICER is recalculated.

Table 3. Computation of ICER after dropping strategy III.

\begin{tabular}{llll}
\hline Strategy & Total Cost(\$) & TotalInfections Averted & ICER \\
\hline Strategy II & $\$ 2,770.20$ & $75,510.00$ & 0.0367 \\
Strategy I & $\$ 3,090.00$ & $91,109.00$ & 0.0205 \\
Strategy IV & $\$ 4,383.30$ & $93,870.00$ & 0.4684 \\
\hline
\end{tabular}

Comparing strategy II and strategy I, ICER of strategy I is less than ICER of strategy II. Thus, strategy II is omitted and the ICER is recalculated.

Table 4. Computation of ICER after dropping strategy II.

\begin{tabular}{llll}
\hline Strategy & Total Cost(\$) & TotalInfections Averted & ICER \\
\hline Strategy I & $\$ 3,090.00$ & $91,109.00$ & 0.0339 \\
Strategy IV & $\$ 4,383.30$ & $93,870.00$ & 0.4684 \\
\hline
\end{tabular}

By comparing strategy I and strategy IV, ICER of strategy I is less than ICER of strategy IV. Therefore, strategy IV is dropped and strategy I is considered.

Thus, according to Incremental Cost Effectiveness Ratio analysis, the combination of optimal prevention and treatment control strategies is the best way of minimizing cervical cancer among women with or without HIV infection in our community following the combination of all control strategies.

\section{Conclusion}

This paper designed and analyzed a deterministic model for co-infection of cervical cancer and HIV diseases. The optimal control theory was employed to the main model and analyzed using Potrayagin's Maximum Principle. The different combination of three optimal control strategies; prevention, screening, and treatment were studied numerically. Also, cost-effectiveness analysis was performed and the following results were obtained

(a) The combination of optimal screening and treatment strategies is not much powerful way of controlling HPV infection and cervical cancer in the community compared with other combination of optimal control strategies as shown in Figure 3. Also, applying the combination of all control strategies (prevention, screening, and treatment) is the best way of minimizing co-infection of cervical cancer and HIV diseases in a community as shown in Figure 4.

(b) In the case of Incremental Cost Effectiveness Ratio analysis, the combination of optimal prevention and treatment strategies is the most cost-effective control to minimize cervical cancer among women with or without HIV infection. The second is the combination of all optimal control strategies; prevention, screening, and treatment. The third is the combination of optimal prevention and screening control strategies.

\section{References}

[1] S. E. Hawes, C. W. Critchlow, M. A. Faye Niang, M. B. Diouf, A. Diop, P. Toure, A. Aziz Kasse, B. Dembele, P. Salif Sow, A. M. Coll-Seck, J. M. Kuypers, N. B. Kiviat, H. S. E., C. C. W., F. N. M. A., D. M. B., D. A., T. P., K. A. A., D. B., S. P. S., C.-S. A. M., K. J. M., and K. N. B., "Increased risk of high-grade cervical squamous intraepithelial lesions and invasive cervical cancer among African women with human immunodeficiency virus type 1 and 2 infections,"J. Infect. Dis., 2003, vol. 188, no. 4, pp. 555-563.

[2] S. M. Mbulaiteye, E. T. Katabira, H. Wabinga, D. M. Parkin, P. Virgo, R. Ochai, M. Workneh, A. Coutinho, and E. A. Engels, "Spectrum of cancers among HIV-infected persons in Africa: The Uganda AIDS-Cancer registry match study," Int. J. Cancer, 2006, vol. 118, no. 4, pp. 985-990.

[3] C. Ng'andwe, J. J. Lowe, P. J. Richards, L. Hause, C. Wood, and P. C. Angeletti, "The distribution of sexually-transmitted human papillomaviruses in HIV positive and negative patients in Zambia, Africa.," BMC Infect. Dis., 2007, vol. 7, pp. 77.

[4] B. Maregere, "Analysis of co-infection of human immunodeficiency virus with human papillomavirus," University of KwaZulu-Natal, 2014.

[5] K. O. Okosun and O. D. Makinde, "A co-infection model of malaria and cholera diseases with optimal control," Math. Biosci., 2014, vol. 258, pp. 19-32.

[6] K. O. Okosun and O. D. Makinde, "Optimal control analysis of malaria in the presence of non-linear incidence rate," Appl. Comput. Math., 2013, vol. 12, no. 1, pp. 20-32.

[7] K. O. Okosun, O. D. Makinde, and I. Takaidza, "Analysis of recruitment and industrial human resources management for optimal productivity in the presence of the HIV/AIDS epidemic," J. Biol. Phys., 2013, vol. 39, no. 1, pp. 99-121.

[8] S. Lenhart and J. T. Workman, Optimal control applied to biological models dynamic optimization, 2007.

[9] W. H. Fleming and R. W. Rishel, Deterministic and stochastic optimal control, Springer-Verlag, Berlin Heidelberg New York, 1975.

[10] K. O. Okosun, O. D. Makinde, and I. Takaidza, "Impact of optimal control on the treatment of HIV/AIDS and screening of unaware infectives," Appl. Math. Model., 2013, vol. 37, no. 6, pp. 3802-3820.

[11] "Tanzania-life expectance at birth." [Online]. Available: http://countryeconomy.com/demography/lifeexpectancy/tanzania. [Accessed: 09-Oct-2016].

[12] S. L. Lee and A. M. Tameru, "A mathematical model of human papillomavirus (HPV) in the united states and its impact on cervical cancer," J. Cancer, 2012, vol. 3, no. 1, pp. 262-268.

[13] R. Federation, S. Africa, and S. Lanka, "Cervical cancer global crisis card," Cerv. Cancer Free Coalit., 2013.

[14] "HIV and AIDS in Tanzania," 2015. [Online]. Available: http://www.avert.org/professionals/hiv-around-world/subsaharan-africa/tanzania. [Accessed: 09-Oct-2016].

[15] G. G. Sanga, O. D. Makinde, E. S. Massawe and L. Namkinga, "Modelling co-dynamics of cervical cancer and HIV diseases, Glob. J. Appl. Math., 2017, vol. 13, no. 6, pp. 2057-2078. 